\title{
The boundary states and correlation functions of the tricritical Ising model from the Coulomb-gas formalism.
}

\author{
Smain BALASKA*and Toufik SAHABI ${ }^{\dagger}$ \\ Laboratoire de physique théorique d'Oran. \\ Département de physique. Université d'Oran Es-Sénia. \\ 31100 Es-Sénia. ALGERIA
}

September 20, 2018

\begin{abstract}
We consider the minimal conformal model describing the tricritical Ising model on the disk and on the upper half plane. Using the coulomb-gas formalism we determine its consistents boundary states as well as its 1-point and 2-point correlation functions.
\end{abstract}

PACS numbers : 11.25.Hf

\section{Introduction}

The basic concepts and techniques of BCFT were introduced first by J. L. Cardy. He studied in [1] how to restrict the operator content by imposing the boundary conditions. He has obtained a classification of the boundary states and has developed a method to calculate the boundary correlation functions ([2], [3] and [4]).

An alternative approach to obtain correlation functions for Cardy's boundary states is developed in [5] and [6]. It's a reformulation of the known Coulomb-gas formalism for theories with boundaries. Instead of using the contour integration technique (as this was done in [7]) the author constructs the representation of the boundary states on the charged bosonic Fock space

*e-mail : sbalaska@yahoo.com, balaska.smain@univ-oran.dz

${ }^{\dagger}$ e-mail : sahabitoufik@yahoo.fr 
and uses the charge neutrality conditions to obtain the correct linear combination of the conformal blocks in the expression of the correlation functions. This formalism provides a method for calculating correlation functions in the case of minimal conformal field theories without having to solve differential equations.

Using this formalism we study the particular case of the minimal conformal model $\left(A_{4}, A_{3}\right)$ on the upper half plane. The boundary one-point and two-points correlators of this model are calculated. The supersymmetric version of the boundary tricritical Ising field theory is studied in [8, [9] and [10].

The paper is organised as follows. In section 2, we start by considering the general case of a conformal theory defined on the upper half plane and study the consequences of the existence of boundaries on the conformal algebra and on the structure of its Hilbert space. In section 3, using the Cardy's fusion method [2] we derive a classification of the conformal theories one can define on the bounded geometry in terms of the boundary conditions. In section 4 we determine the consistent boudary states as well as the partition functions for the particular case of the minimal model $\left(A_{4}, A_{3}\right)$.

In section 5, we give the essential of the method used in [5] and [6] and we apply it to determine the one-point as well as the two-point correlators of the tricritical Ising model on the disk and the upper half plane.

\section{A CFT on the Upper Half plane}

Let us start with a CFT on the upper half plane (UHP). We define $z=x+i y$ and we consider a theory defined on the region $\operatorname{Im}(z) \geq 0$.

In this case only real analytic changes of coordinates, with

$$
\varepsilon(z)=\left.\bar{\varepsilon}(\bar{z})\right|_{z=\bar{z} \in R}
$$

are allowed. The energy momentum verify

$$
T(z)=\left.\bar{T}(\bar{z})\right|_{\text {real axis }}
$$

so that there is no momentum flow across the boundary. There is thus only one copy of the Virasoro algebra

$$
\bar{L}_{-n}=\left.L_{n}\right|_{z=\bar{z}}
$$

Boundary conditions labelled by $\alpha$ and $\beta$ (for $\operatorname{Re}(z) \prec 0$ and $\operatorname{Re}(z) \succ 0$ ) are assigned to fields on the boundary. The UHP can conformally mapped on an infinite horizontal strip of width $L$ by $w=\frac{L}{\pi} \ln z$ 
In both geometries, the system is described by a Hilbert space of states $\mathcal{H}_{\alpha \mid \beta}$ which decomposes on representations $V_{i}$ of the Virasoro algebra according to

$$
\mathcal{H}_{\alpha \mid \beta}=\oplus n_{i \alpha}{ }^{\beta} V_{i}
$$

where the integers $n_{i \alpha}{ }^{\beta}$ are the multiplicities of the representations.

On the strip, the Hamiltonian is the translation operator in the direction $\operatorname{Re}(w)$. It can be written in the UHP as

$$
H_{\alpha \beta}=\frac{2 \pi}{L}\left(L_{0}-\frac{c}{24}\right)
$$

To determine the operator content of the theory, we need to determine the possible boundary conditions $\alpha, \beta$ on the UHP and also the associated multiplicities $n_{i \alpha}{ }^{\beta}$

\section{The physical boundary states and the par- tition functions}

We proceed as in the case of the theories defined on the tore. We take a semi annular domain in the half plane and identify its edges to make a cylinder. Equivalently one can consider instead a finite strip. In fact the semi annular domain comprised between the semi-circles of radius 1 and $e^{\frac{\pi T}{L}}$ (in the $z$ UHP) can be mapped in the $w$ plane into the segment $0 \leq \operatorname{Re}(w) \leq T$ of the strip of width $L$ by the transformation $w(z)=\frac{L}{\pi} \ln (z)$. In the same way the latter can also be transformed into the annulus of radius 1 and $e^{\frac{2 \pi L}{T}}=\widetilde{q}^{-1 / 2}$ by the transformation $\zeta(w)=\exp (-2 i \pi w / T)\left(\right.$ with $\left.\widetilde{q}=e^{2 \pi i \tau}, \widetilde{\tau}=2 i T / L\right)$.

The annulus in the $\zeta$ plane is equivalent to a finite cylinder of length $T$ and circumference $L$. When considering such geometry one is allowed to use the familiar energy momentum tensor of the full plane without modification. Then radial quantization is allowed and the conformal invariance condition (2) on the quantum states $|\alpha\rangle$ is [2]

$$
\left(L_{n}^{\zeta}-\bar{L}_{-n}^{\zeta}\right)|\alpha\rangle=0
$$

There is a basis of states, which are solution of this linear system of boundary conditions. It is the basis of Ishibashi states [11]. There exists an independent Ishibashi states (noted $|j\rangle\rangle$ ) solution of (6) for each representation $V_{j}$ of the algebra. The equation (6) being linear, any linear combination of the Ishibashi states is also a solution. To obtain the combinations corresponding to the physical boundary states, one uses the Cardy's fusion method [2] (for review see also [12], [13]) 
It consists in calculating the partition function $\mathcal{Z}_{\alpha \mid \beta}$ in two different ways. First as resulting from the evolution between the boundary states $\langle\alpha|$ and $|\beta\rangle$

$$
\begin{aligned}
\mathcal{Z}_{\alpha \mid \beta} & =\left\langle\alpha\left|e^{-T H}\right| \beta\right\rangle \\
& =\left\langle\alpha\left|\widetilde{q}^{\frac{1}{2}\left(L_{0}+\bar{L}_{0}-\frac{c}{12}\right)}\right| \beta\right\rangle
\end{aligned}
$$

where $H=\frac{2 \pi}{L}\left(L_{0}+\bar{L}_{0}-\frac{c}{12}\right)$ and $\widetilde{q}=\exp \left(-4 \pi \frac{T}{L}\right)$

On the other hand using the decomposition (4) of the hilbert space $\mathcal{H}_{\alpha \mid \beta}$ one obtain

$$
\mathcal{Z}_{\alpha \mid \beta}(q)=\sum_{j} n_{j \alpha}{ }^{\beta} \chi_{j}(q)
$$

where $\chi_{j}(q)$ is the character of the representation $V_{j}$ and $q=e^{-\pi L / T}$

Expanding the physical boundary states as

$$
\left.|\alpha\rangle=\sum_{j} \frac{a_{\alpha j}}{\sqrt{S_{1 j}}}|j\rangle\right\rangle
$$

$S_{i j}$ being the element of the matrix $S$ of the modular group and equating (7) and (8), we obtain

$$
\left.\sum_{j} n_{j \alpha}^{\beta} \chi_{j}(q)=\sum_{j j^{\prime}}\langle\alpha \mid j\rangle\right\rangle\left\langle\left\langle j\left|\widetilde{q}^{\frac{1}{2}\left(L_{0}+\bar{L}_{0}-\frac{c}{12}\right)}\right| j^{\prime}\right\rangle\right\rangle\left\langle\left\langle j^{\prime} \mid \beta\right\rangle\right.
$$

The Ishibashi states are so that

$$
\left\langle\left\langle j\left|\widetilde{q}^{\frac{1}{2}\left(L_{0}+\bar{L}_{0}-\frac{c}{12}\right)}\right| j^{\prime}\right\rangle\right\rangle=\delta_{j j^{\prime}} \chi_{j}(\widetilde{q})
$$

then (10) becomes

$$
\sum_{j} n_{j \alpha}{ }^{\beta} \chi_{j}(q)=\sum_{j} \frac{a_{\alpha j}^{*} a_{\beta j}}{S_{1 j}} \chi_{j}(\widetilde{q})
$$

Now performing a modular transformation on the characters

$$
\chi_{j}(\widetilde{q})=\sum_{i} S_{i j} \chi_{i}(q)
$$

and identifying the coefficients of $\chi_{i}$, one obtains

$$
n_{j \alpha}{ }^{\beta}=\sum_{i} \frac{S_{j i} a_{\alpha i}^{*} a_{\beta i}}{S_{1 i}}
$$


To impose the orthonormality condition $\langle\alpha \mid \beta\rangle=\delta_{\alpha \beta}$, the coefficients $a_{\alpha j}$ have to verify

$$
\sum_{j} a_{\alpha j}^{*} a_{\beta j}=\delta_{\alpha \beta}
$$

As a solution, one can take (recall that the matrix $S$ is unitary)

$$
a_{i j}=S_{i j}
$$

so that finally we have for the physical (or what we also call consistent) boundary states

$$
\left.|\alpha\rangle=\sum_{j} \frac{S_{\alpha j}}{\sqrt{S_{1 j}}}|j\rangle\right\rangle
$$

and for the multiplicities

$$
n_{j \alpha}{ }^{\beta}=\sum_{i} \frac{S_{j i} S_{\alpha j}^{*} S_{\beta j}}{S_{1 i}}
$$

This equation is no more than the Verlinde formula [14]. It gives the fusion multiplicities appearing into the decomposition of the fusion of two representations of the algebra in terms of the elements of the $S$ matrix.

Thus the multiplicities $n_{i \alpha}{ }^{\beta}$ are identified with the coefficients of the fusion algebra

$$
n_{i \alpha}{ }^{\beta}=N_{i \alpha}{ }^{\beta}
$$

We obtain then a classification of the different conformal field theories on the upper half plane and their operator contents. The later depends on the boundary conditions.

\section{The case of the tricritical ising model $\left(A_{4}, A_{3}\right)$ :}

The central charge, in this case, is $c=7 / 10$, and the Kac table contents six primary fields indexed by the pairs $(r, s)$ given in the set $\varepsilon$. See table (1) for operator contents of the model.

$$
\varepsilon=\left\{\begin{array}{c}
(1,1)=(3,4),(1,2)=(3,3),(1,3)=(3,2) \\
(1,4)=(3,1),(2,2)=(2,3),(2,4)=(2,1)
\end{array}\right\}
$$

The modular matrix $S$ is given by 


\begin{tabular}{||c||c||c||}
\hline$(r, s)$ & Dimension & signe \\
\hline$(1,1)$ or $(3,4)$ & 0 & $I$ \\
\hline$(1,2)$ or $(3,3)$ & $1 / 10$ & $\varepsilon$ \\
\hline$(1,3)$ or $(3,2)$ & $3 / 5$ & $\varepsilon^{\prime}$ \\
\hline$(1,4)$ or $(3,1)$ & $3 / 2$ & $\varepsilon^{\prime \prime}$ \\
\hline$(2,2)$ or $(2,3)$ & $3 / 80$ & $\sigma$ \\
\hline$(2,4)$ or $(2,1)$ & $7 / 16$ & $\sigma^{\prime}$ \\
\hline
\end{tabular}

Table 1: The primary fields of the tricritical Ising model.

$$
S=\frac{1}{\sqrt{5}}\left(\begin{array}{cccccc}
s_{2} & s_{1} & s_{1} & s_{2} & \sqrt{2} s_{1} & \sqrt{2} s_{2} \\
s_{1} & -s_{2} & -s_{2} & s_{1} & \sqrt{2} s_{2} & -\sqrt{2} s_{1} \\
s_{1} & -s_{2} & -s_{2} & s_{1} & -\sqrt{2} s_{2} & \sqrt{2} s_{1} \\
s_{2} & s_{1} & s_{1} & s_{2} & -\sqrt{2} s_{1} & -\sqrt{2} s_{2} \\
\sqrt{2} s_{1} & \sqrt{2} s_{2} & -\sqrt{2} s_{2} & -\sqrt{2} s_{1} & 0 & 0 \\
\sqrt{2} s_{2} & -\sqrt{2} s_{1} & \sqrt{2} s_{1} & -\sqrt{2} s_{2} & 0 & 0
\end{array}\right)
$$

with $s_{1}=\sin 2 \pi / 5$, and $s_{2}=\sin 4 \pi / 5$.

\subsection{The physical boundary states}

As we already mentioned for the diagonal minimal models of type $\left(A_{p-1}, A_{p^{\prime}-1}\right)$, the coefficients $a_{\alpha}^{j}$ introduced in equation (91) are equal to the elements of the matrix $S$ [13]. Then the Consistent boundary states for this model are written as

$$
\left.|(r, s)\rangle=\sum_{\left(r^{\prime}, s^{\prime}\right) \in \varepsilon} \frac{S_{r s, r^{\prime} s^{\prime}}}{\sqrt{S_{11, r^{\prime} s^{\prime}}}}\left|\left(r^{\prime}, s^{\prime}\right)\right\rangle\right\rangle
$$

These are the six states given in the following table

\subsection{The fusion rules and the partition functions}

The fusion between the different operators of the model are resumed as follows 


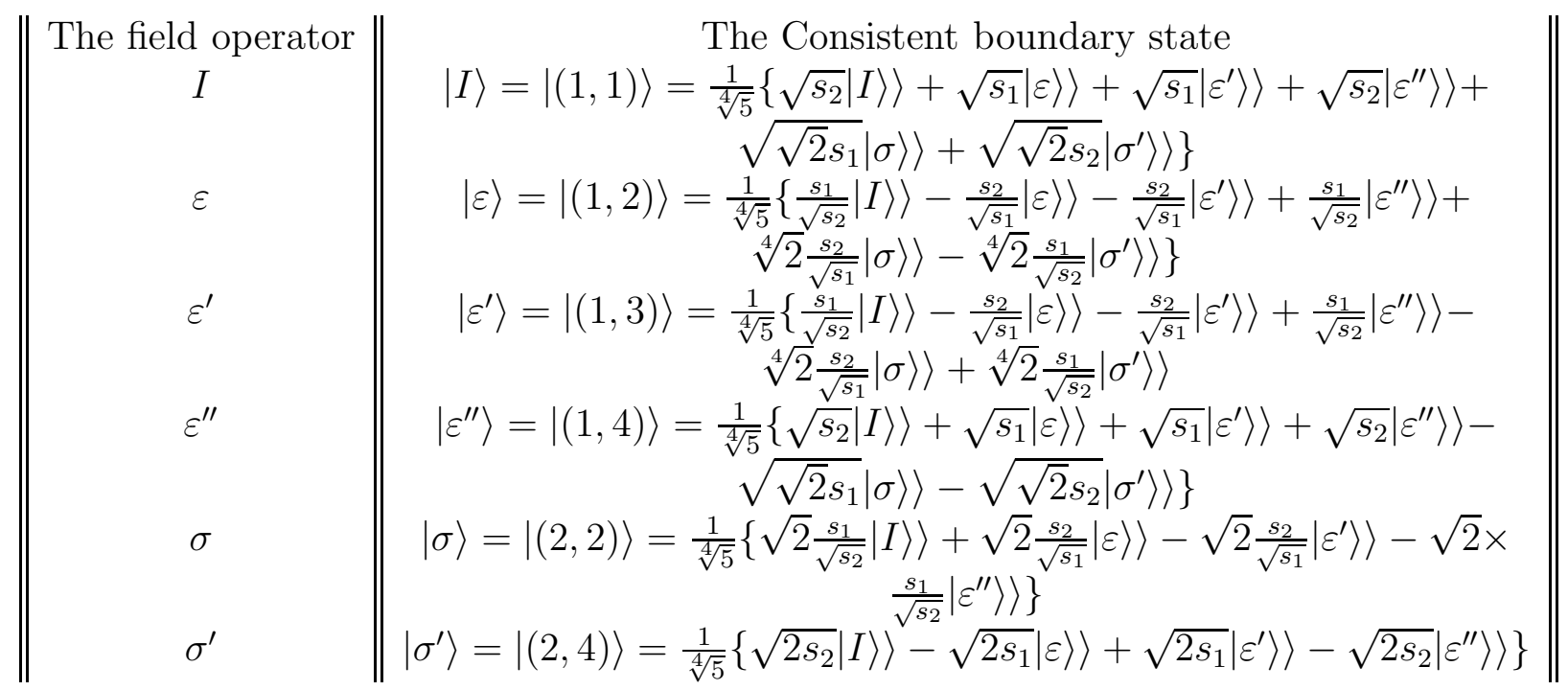

Table 2: The Consistent boundary states of the tricritical Ising model

$$
\begin{gathered}
\left(\begin{array}{lllllll}
I & \varepsilon & \varepsilon^{\prime} & \varepsilon^{\prime \prime} & \sigma & \sigma^{\prime} \\
\varepsilon & \varepsilon^{2} & \varepsilon \varepsilon^{\prime} & \varepsilon \varepsilon^{\prime \prime} & \varepsilon \sigma & \varepsilon \sigma^{\prime} \\
\varepsilon^{\prime} & \varepsilon^{\prime} \varepsilon & \varepsilon^{\prime 2} & \varepsilon^{\prime} \varepsilon^{\prime \prime} & \varepsilon^{\prime} \sigma & \varepsilon^{\prime} \sigma^{\prime} \\
\varepsilon^{\prime \prime} & \varepsilon^{\prime \prime} \varepsilon & \varepsilon^{\prime \prime} \varepsilon^{\prime} & \varepsilon^{\prime \prime 2} & \varepsilon^{\prime \prime} \sigma & \varepsilon^{\prime \prime} \sigma^{\prime} \\
\sigma & \sigma \varepsilon & \sigma \varepsilon^{\prime} & \sigma \varepsilon^{\prime \prime} & \sigma^{2} & \sigma \sigma^{\prime} \\
\sigma^{\prime} & \sigma^{\prime} \varepsilon & \sigma^{\prime} \varepsilon^{\prime} & \sigma^{\prime} \varepsilon^{\prime \prime} & \sigma^{\prime} \sigma & \sigma^{\prime 2}
\end{array}\right)= \\
=\left(\begin{array}{llllll}
1 & 0 & 0 & 0 & 0 & 0 \\
0 & 1 & 0 & 0 & 0 & 0 \\
0 & 0 & 1 & 0 & 0 & 0 \\
0 & 0 & 0 & 1 & 0 & 0 \\
0 & 0 & 0 & 0 & 1 & 0 \\
0 & 0 & 0 & 0 & 0 & 1
\end{array}\right) I+\left(\begin{array}{lllllll}
0 & 1 & 0 & 0 & 0 & 0 \\
1 & 0 & 1 & 0 & 0 & 0 \\
0 & 1 & 0 & 1 & 0 & 0 \\
0 & 0 & 1 & 0 & 0 & 0 \\
0 & 0 & 0 & 0 & 1 & 1 \\
0 & 0 & 0 & 0 & 1 & 0
\end{array}\right) \varepsilon \\
+\left(\begin{array}{llllll}
0 & 0 & 1 & 0 & 0 & 0 \\
0 & 1 & 0 & 1 & 0 & 0 \\
1 & 0 & 1 & 0 & 0 & 0 \\
0 & 1 & 0 & 0 & 0 & 0 \\
0 & 0 & 0 & 0 & 1 & 1 \\
0 & 0 & 0 & 0 & 1 & 0
\end{array}\right) \varepsilon^{\prime}+\left(\begin{array}{llllll}
0 & 0 & 0 & 1 & 0 & 0 \\
0 & 0 & 1 & 0 & 0 & 0 \\
0 & 1 & 0 & 0 & 0 & 0 \\
1 & 0 & 0 & 0 & 0 & 0 \\
0 & 0 & 0 & 0 & 1 & 0 \\
0 & 0 & 0 & 0 & 0 & 1
\end{array}\right) \varepsilon^{\prime \prime}
\end{gathered}
$$




$$
+\left(\begin{array}{llllll}
0 & 0 & 0 & 0 & 1 & 0 \\
0 & 0 & 0 & 0 & 1 & 1 \\
0 & 0 & 0 & 0 & 1 & 1 \\
0 & 0 & 0 & 0 & 1 & 0 \\
1 & 1 & 1 & 1 & 0 & 0 \\
0 & 1 & 1 & 0 & 0 & 0
\end{array}\right) \sigma+\left(\begin{array}{llllll}
0 & 0 & 0 & 0 & 0 & 1 \\
0 & 0 & 0 & 0 & 1 & 0 \\
0 & 0 & 0 & 0 & 1 & 0 \\
0 & 0 & 0 & 0 & 0 & 1 \\
0 & 1 & 1 & 0 & 0 & 0 \\
1 & 0 & 0 & 1 & 0 & 0
\end{array}\right) \sigma^{\prime}
$$

Using the equations (8) and (19) we obtain the twelve following partition functions

$$
\begin{aligned}
\mathcal{Z}_{I \mid I} & =\mathcal{Z}_{\varepsilon^{\prime \prime} \mid \varepsilon^{\prime \prime}}=\chi_{1,1}(q), \\
\mathcal{Z}_{I \mid \varepsilon} & =\mathcal{Z}_{\varepsilon^{\prime} \mid \varepsilon^{\prime \prime}}=\chi_{1,2}(q), \\
\mathcal{Z}_{I \mid \varepsilon^{\prime}} & =\mathcal{Z}_{\varepsilon \mid \varepsilon^{\prime \prime}}=\chi_{1,3}(q), \\
\mathcal{Z}_{I \mid \varepsilon^{\prime \prime}} & =\chi_{1,4}(q) \\
\mathcal{Z}_{I \mid \sigma} & =\mathcal{Z}_{\varepsilon \mid \sigma^{\prime}}=\mathcal{Z}_{\varepsilon^{\prime} \mid \sigma^{\prime}}=\mathcal{Z}_{\varepsilon^{\prime \prime} \mid \sigma}=\chi_{2,2}(q), \\
\mathcal{Z}_{I \mid \sigma^{\prime}} & =\mathcal{Z}_{\varepsilon^{\prime \prime} \mid \sigma^{\prime}}=\chi_{2,4}(q), \\
\mathcal{Z}_{\varepsilon \mid \varepsilon} & =\mathcal{Z}_{\varepsilon^{\prime} \mid \varepsilon^{\prime}}=\chi_{1,1}(q)+\chi_{1,3}(q), \\
\mathcal{Z}_{\varepsilon \mid \varepsilon^{\prime}} & =\chi_{1,2}(q)+\chi_{1,4}(q), \\
\mathcal{Z}_{\varepsilon \mid \sigma} & =\mathcal{Z}_{\varepsilon^{\prime} \mid \sigma}=\chi_{2,2}(q)+\chi_{2,4}(q), \\
\mathcal{Z}_{\sigma \mid \sigma} & =\chi_{1,1}(q)+\chi_{1,2}(q)+\chi_{1,3}(q)+\chi_{1,4}(q), \\
\mathcal{Z}_{\sigma \mid \sigma^{\prime}} & =\chi_{1,2}(q)+\chi_{1,3}(q), \\
\mathcal{Z}_{\sigma^{\prime} \mid \sigma^{\prime}} & =\chi_{1,1}(q)+\chi_{1,4}(q)
\end{aligned}
$$

where we have taken into account the fact that

$$
\mathcal{Z}_{\alpha \mid \beta}=\mathcal{Z}_{\beta \mid \alpha}
$$

\section{The boundary correlation functions}

\subsection{The coulomb gas formalism and the coherent bound- ary states}

In the coulomb gas-formalism one reproduces the primary fields $\phi_{r, s}(0<$ $\left.r<p, 0<s<p^{\prime}\right)$ of the Kac table of the minimal model $\left(A_{p-1,} A_{p^{\prime}-1}\right)$ by the vertex operators

$$
\mathcal{V}_{\alpha_{r, s}}(z)=: \exp \left(i \sqrt{2} \alpha_{r, s} \varphi(z):\right.
$$


with conformal dimensions given by

$$
h_{r, s}=\frac{1}{4}\left(r \alpha_{+}+s \alpha_{-}\right)^{2}-\alpha_{0}^{2}
$$

where $\varphi(z)$ is the holomorphic part of a boson field $\Phi(z, \bar{z})$ and

$$
\begin{aligned}
\alpha_{r, s} & =\frac{1}{2}(1-r) \alpha_{+}+\frac{1}{2}(1-s) \alpha_{-} \\
\alpha_{+} & =\sqrt{p / p^{\prime}} \\
\alpha_{-} & =-\sqrt{p^{\prime} / p}
\end{aligned}
$$

The coefficient $\alpha_{0}$ is related to the central charge by

$$
c=1-24 \alpha_{0}^{2}
$$

It is known (see for example [15]) that the correlation function of $n$ vertex operators is equal to

$$
\left\langle\prod_{i=1}^{n} \mathcal{V}_{\alpha_{i}}\left(z_{i}\right)\right\rangle=\left\{\begin{array}{ccc}
\prod_{i<j}^{n}\left(z_{i}-z_{j}\right)^{2 \alpha_{i} \alpha_{j}} & \text { if } & \sum_{i=1}^{n} \alpha_{i}=2 \alpha_{0} \\
0 & \text { otherwise }
\end{array}\right\}
$$

The fock space $F_{\alpha, \alpha_{0}}$ related to the vertex operator $\mathcal{V}_{\alpha}$ is built on the highest weight state

$$
\left|\alpha, \alpha_{0}\right\rangle=\exp \left(i \sqrt{2} \alpha \widehat{\varphi}_{0}\right)\left|0, \alpha_{0}\right\rangle
$$

by the action of the mode operators $\widehat{a}_{m}$ of the field $\varphi(z)$, defined by

$$
\varphi(z)=\widehat{\varphi_{0}}-i \widehat{a}_{0} \ln (z)+i \sum_{n \neq 0} \frac{\widehat{a}_{n}}{n} z^{-n}
$$

and satisfying the algebra

$$
\begin{aligned}
{\left[\widehat{a}_{m}, \widehat{a}_{n}\right] } & =m \delta_{m+n} \\
{\left[\widehat{\varphi}_{0}, \widehat{a}_{0}\right] } & =i
\end{aligned}
$$

The state $\left|0, \alpha_{0}\right\rangle$ is the vacuum state and we have

$$
\begin{gathered}
\widehat{a}_{0}\left|\alpha, \alpha_{0}\right\rangle=\sqrt{2} \alpha\left|\alpha, \alpha_{0}\right\rangle \\
\left\langle\alpha, \alpha_{0} \mid \beta, \alpha_{0}\right\rangle=\kappa \delta_{\alpha \beta}
\end{gathered}
$$

$\kappa$ being a normalization constant which can be chosen to be equal to one. 
The aim goal now is to construct conformally boundary states in terms of the states $\left|\alpha, \alpha_{0}\right\rangle$ of the different fock spaces corresponding to the different values of $\alpha$. Starting from the ansatz

$$
\left|B_{\alpha, \bar{\alpha}, \alpha_{0}}\right\rangle=\prod_{k>0} \exp \left(-\frac{1}{k} \widehat{a}_{-k} \widehat{\bar{a}}_{-k}\right)\left|\alpha, \bar{\alpha}, \alpha_{0}\right\rangle
$$

where the states $\left|\alpha, \bar{\alpha}, \alpha_{0}\right\rangle$ are the direct product of the holomorphic and antiholomorphic highest weight states

$$
\left|\alpha, \bar{\alpha}, \alpha_{0}\right\rangle=\left|\alpha, \alpha_{0}\right\rangle \otimes\left|\bar{\alpha}, \alpha_{0}\right\rangle
$$

and by expressing the elements of the Virasoro algebra in terms of the operators $\widehat{a}_{m}$, one can easily show that the states $\left|B_{\alpha, \bar{\alpha}, \alpha_{0}}\right\rangle$ verify

$$
\left(L_{n}-\bar{L}_{-n}\right)\left|B_{\alpha, \bar{\alpha}, \alpha_{0}}\right\rangle=0
$$

only if

$$
\alpha+\bar{\alpha}-2 \alpha_{0}=0
$$

The boundary states satisfying these two conditions will be noted

$$
\left|B_{\alpha, \bar{\alpha}, \alpha_{0}}\right\rangle=\left|B_{\alpha, 2 \alpha_{0}-\alpha, \alpha_{0}}\right\rangle=|B(\alpha)\rangle
$$

In addition to the necessary condition (34), the coherent states $|B(\alpha)\rangle$ have to satisfy the conditions obtained from the duality of the partition function as already done in section 1 . There is in fact a combination of these states which satisfy the duality condition and it is shown in [5] that the Ishibashi states are related to these states as

$$
|(r, s)>>=| a_{r, s}>+\mid a_{r,-s}>
$$

with

$$
\left|a_{r, s}>=\sum_{k \in Z}\right| B\left(k \sqrt{p p^{\prime}}+\alpha_{r, s}\right)>
$$

Using (37) and (17) one can write the physical (or consistent) boundary states of any minimal model in terms of the coherent ones. For the particular case of the minimal model $\left(A_{4}, A_{3}\right)$ we have the following relations

$$
\begin{aligned}
|I\rangle= & \frac{1}{\sqrt{2}} \frac{1}{\sqrt[4]{5}}\left\{\sqrt{s_{2}}\left(\left|a_{1,1}\right\rangle+\left|a_{1,-1}\right\rangle+\left|a_{3,4}\right\rangle+\left|a_{3,-4}\right\rangle\right)+\sqrt{s_{1}}\left(\left|a_{1,2}\right\rangle+\right.\right. \\
& \left.\left|a_{1,-2}\right\rangle+\left|a_{3,3}\right\rangle+\left|a_{3,-3}\right\rangle\right)+\sqrt{s_{1}}\left(\left|a_{1,3}\right\rangle+\left|a_{1,-3}\right\rangle+\left|a_{3,2}\right\rangle+\left|a_{3,-2}\right\rangle\right)+ \\
& \sqrt{s_{2}}\left(\left|a_{1,4}\right\rangle+\left|a_{1,-4}\right\rangle+\left|a_{3,1}\right\rangle+\left|a_{3,-1}\right\rangle\right)+\sqrt{\sqrt{2} s_{1}}\left(\left|a_{2,2}\right\rangle+\left|a_{2,-2}\right\rangle+\right. \\
& \left.\left.\left|a_{2,3}\right\rangle+\left|a_{2,-3}\right\rangle\right)+\sqrt{\sqrt{2} s_{2}}\left(\left|a_{2,4}\right\rangle+\left|a_{2,-4}\right\rangle+\left|a_{2,1}\right\rangle+\left|a_{2,-1}\right\rangle\right)\right\}
\end{aligned}
$$




$$
\begin{aligned}
|\varepsilon\rangle= & \frac{1}{\sqrt{2}} \frac{1}{\sqrt[4]{5}}\left\{\frac{s_{1}}{\sqrt{s_{2}}}\left(\left|a_{1,1}\right\rangle+\left|a_{1,-1}\right\rangle+\left|a_{3,4}\right\rangle+\left|a_{3,-4}\right\rangle\right)-\frac{s_{2}}{\sqrt{s_{1}}}\left(\left|a_{1,2}\right\rangle+\right.\right. \\
& \left.\left|a_{1,-2}\right\rangle+\left|a_{3,3}\right\rangle+\left|a_{3,-3}\right\rangle\right)-\frac{s_{2}}{\sqrt{s_{1}}}\left(\left|a_{1,3}\right\rangle+\left|a_{1,-3}\right\rangle+\left|a_{3,2}\right\rangle+\left|a_{3,-2}\right\rangle\right)+ \\
& \frac{s_{1}}{\sqrt{s_{2}}}\left(\left|a_{1,4}\right\rangle+\left|a_{1,-4}\right\rangle+\left|a_{3,1}\right\rangle+\left|a_{3,-1}\right\rangle\right)+\sqrt[4]{2} \frac{s_{2}}{\sqrt{s_{1}}}\left(\left|a_{2,2}\right\rangle+\left|a_{2,-2}\right\rangle+\right. \\
& \left.\left.\left|a_{2,3}\right\rangle+\left|a_{2,-3}\right\rangle\right)-\sqrt[4]{2} \frac{s_{1}}{\sqrt{s_{2}}}\left(\left|a_{2,4}\right\rangle+\left|a_{2,-4}\right\rangle+\left|a_{2,1}\right\rangle+\left|a_{2,-1}\right\rangle\right)\right\} \\
\left|\varepsilon^{\prime}\right\rangle= & \frac{1}{\sqrt{2}} \frac{1}{\sqrt[4]{5}}\left\{\frac{s_{1}}{\sqrt{s_{2}}}\left(\left|a_{1,1}\right\rangle+\left|a_{1,-1}\right\rangle+\left|a_{3,4}\right\rangle+\left|a_{3,-4}\right\rangle\right)-\frac{s_{2}}{\sqrt{s_{1}}}\left(\left|a_{1,2}\right\rangle+\right.\right. \\
& \left.\left|a_{1,-2}\right\rangle+\left|a_{3,3}\right\rangle+\left|a_{3,-3}\right\rangle\right)-\frac{s_{2}}{\sqrt{s_{1}}}\left(\left|a_{1,3}\right\rangle+\left|a_{1,-3}\right\rangle+\left|a_{3,2}\right\rangle+\left|a_{3,-2}\right\rangle\right)+ \\
& \frac{s_{1}}{\sqrt{s_{2}}}\left(\left|a_{1,4}\right\rangle+\left|a_{1,-4}\right\rangle+\left|a_{3,1}\right\rangle+\left|a_{3,-1}\right\rangle\right)-\sqrt[4]{2} \frac{s_{2}}{\sqrt{s_{1}}}\left(\left|a_{2,2}\right\rangle+\left|a_{2,-2}\right\rangle+\right. \\
& \left.\left.\left|a_{2,3}\right\rangle+\left|a_{2,-3}\right\rangle\right)+\sqrt[4]{2} \frac{s_{1}}{\sqrt{s_{2}}}\left(\left|a_{2,4}\right\rangle+\left|a_{2,-4}\right\rangle+\left|a_{2,1}\right\rangle+\left|a_{2,-1}\right\rangle\right)\right\} \\
\left|\varepsilon^{\prime \prime}\right\rangle= & \frac{1}{\sqrt{2}} \frac{1}{\sqrt[4]{5}}\left\{\sqrt{s_{2}}\left(\left|a_{1,1}\right\rangle+\left|a_{1,-1}\right\rangle+\left|a_{3,4}\right\rangle+\left|a_{3,-4}\right\rangle\right)+\sqrt{s_{1}}\left(\left|a_{1,2}\right\rangle+\right.\right. \\
& \left.\left|a_{1,-2}\right\rangle+\left|a_{3,3}\right\rangle+\left|a_{3,-3}\right\rangle\right)+\sqrt{s_{1}}\left(\left|a_{1,3}\right\rangle+\left|a_{1,-3}\right\rangle+\left|a_{3,2}\right\rangle+\left|a_{3,-2}\right\rangle\right)+ \\
& \sqrt{s_{2}}\left(\left|a_{1,4}\right\rangle+\left|a_{1,-4}\right\rangle+\left|a_{3,1}\right\rangle+\left|a_{3,-1}\right\rangle\right)-\sqrt{\sqrt{2} s_{1}}\left(\left|a_{2,2}\right\rangle+\left|a_{2,-2}\right\rangle+\right. \\
& \left.\left.\left|a_{2,3}\right\rangle+\left|a_{2,-3}\right\rangle\right)-\sqrt{\sqrt{2} s_{2}}\left(\left|a_{2,4}\right\rangle+\left|a_{2,-4}\right\rangle+\left|a_{2,1}\right\rangle+\left|a_{2,-1}\right\rangle\right)\right\} \\
|\sigma\rangle= & \frac{1}{\sqrt[4]{5}}\left\{\frac{s_{1}}{\sqrt{s_{2}}}\left(\left|a_{1,1}\right\rangle+\left|a_{1,-1}\right\rangle+\left|a_{3,4}\right\rangle+\left|a_{3,-4}\right\rangle\right)+\frac{s_{2}}{\sqrt{s_{1}}}\left(\left|a_{1,2}\right\rangle+\right.\right. \\
& \left.\left|a_{1,-2}\right\rangle+\left|a_{3,3}\right\rangle+\left|a_{3,-3}\right\rangle\right)-\frac{s_{2}}{\sqrt{s_{1}}}\left(\left|a_{1,3}\right\rangle+\left|a_{1,-3}\right\rangle+\left|a_{3,2}\right\rangle+\left|a_{3,-2}\right\rangle\right)- \\
& \frac{s_{1}}{\sqrt{s_{2}}}\left(\left|a_{1,4}\right\rangle+\left|a_{1,-4}\right\rangle+\left|a_{3,1}\right\rangle+\left|a_{3,-1}\right\rangle\right) \\
\left|\sigma^{\prime}\right\rangle= & \frac{1}{\sqrt[4]{5}}\left\{\sqrt{s_{2}}\left(\left|a_{1,1}\right\rangle+\left|a_{1,-1}\right\rangle+\left|a_{3,4}\right\rangle+\left|a_{3,-4}\right\rangle\right)-\sqrt{s_{1}}\left(\left|a_{1,2}\right\rangle+\right.\right. \\
& \left.\left|a_{1,-2}\right\rangle+\left|a_{3,3}\right\rangle+\left|a_{3,-3}\right\rangle\right)+\sqrt{s_{1}}\left(\left|a_{1,3}\right\rangle+\left|a_{1,-3}\right\rangle+\left|a_{3,2}\right\rangle+\left|a_{3,-2}\right\rangle\right)- \\
& \sqrt{s_{2}}\left(\left|a_{1,4}\right\rangle+\left|a_{1,-4}\right\rangle+\left|a_{3,1}\right\rangle+\left|a_{3,-1}\right\rangle\right)
\end{aligned}
$$

Note that before using (37), we have replaced each state $|(r, s)\rangle\rangle$ by the symmetrised one

$$
\left.\left.\frac{1}{\sqrt{2}}\{|(r, s)\rangle\rangle+\left|\left(p^{\prime}-r, p-s\right)\right\rangle\right\rangle\right\}
$$

\subsection{The boundary 1-point correlation functions}

In the coulomb gas formalism a boundary p-points correlation function of the form

$$
\left\langle\alpha\left|\phi_{r_{1}, s_{1}}\left(z_{1}, \bar{z}_{1}\right) \phi_{r_{2}, s_{2}}\left(z_{2}, \bar{z}_{2}\right) \ldots \ldots \phi_{r_{p}, s_{p}}\left(z_{p}, \bar{z}_{p}\right)\right| 0\right\rangle
$$

where $|\alpha\rangle$ is one of the allowed physical boundary state for the model, can be written as a combination of correlators having the general form

$$
\left\langle B(\alpha)\left|\prod_{i=1}^{p} \mathcal{V}_{\left(r_{i}, s_{i}\right),\left(\bar{r}_{i}, \bar{s}_{i}\right)}^{\left(m_{i}, n_{i}\right),\left(\bar{n}_{i}, \bar{n}_{i}\right)}\left(z_{i}, \bar{z}_{i}\right)\right| 0,0 ; \alpha_{0}\right\rangle
$$


Where we have noted

$$
\mathcal{V}_{\left(r_{i}, s_{i}\right),\left(\bar{r}_{i}, \bar{s}_{i}\right)}^{\left(m_{i}, \bar{n}_{i}\right)}\left(z_{i}, \bar{z}_{i}\right)=\mathcal{V}_{\left(r_{i}, s_{i}\right)}^{\left(m_{i}, n_{i}\right)}\left(z_{i}\right) \overline{\mathcal{V}}_{\left(\bar{r}_{i}, \bar{s}_{i}\right)}^{\left(\bar{m}_{i}, \bar{n}_{i}\right)}\left(\bar{z}_{i}\right)
$$

with the screened vertex operators

$$
\begin{aligned}
& \mathcal{V}_{(r, s)}^{(m, n)}(z)=\oint \prod_{i=1}^{m} d u_{i} \prod_{j=1}^{n} d v_{j} \mathcal{V}_{r, s}(z) \mathcal{V}_{+}\left(u_{1}\right) \ldots \mathcal{V}_{+}\left(u_{m}\right) \times \mathcal{V}_{-}\left(v_{1}\right) \ldots \mathcal{V}_{-}\left(v_{n}\right), \\
& \overline{\mathcal{V}}_{(\bar{r}, \bar{s})}^{(\bar{m}, \bar{n})}(\bar{z})=\oint \prod_{i=1}^{\bar{m}} d \bar{u}_{i} \prod_{j=1}^{\bar{n}} d \bar{v}_{j} \overline{\mathcal{V}}_{\bar{r}, \bar{s}}(\bar{z}) \mathcal{V}_{+}\left(\bar{u}_{1}\right) \ldots \mathcal{V}_{+}\left(\bar{u}_{\bar{m}}\right) \times \mathcal{V}_{-}\left(\bar{v}_{1}\right) \ldots \mathcal{V}_{-}\left(\bar{v}_{\bar{n}}\right)
\end{aligned}
$$

We recall that the numbers $m$ and $n$ of the screening operators

$$
Q_{ \pm}=\oint d z \mathcal{V}_{\alpha_{ \pm}}(z)=\oint d z \mathcal{V}_{ \pm}(z)
$$

(of conformal dimensions 1 ) is so that the neutrality condition appearing in (29) is fullfiled. For the correlator (40) the neutrality condition for the holomorphic part is

$$
-\alpha+\sum_{i} \alpha_{r_{i}, s_{i}}+\sum_{i} m_{i} \alpha_{+} \sum_{i} n_{i} \alpha_{-}=0
$$

and for the antiholomortphic one, we have

$$
\alpha-2 \alpha_{0}+\sum_{i} \alpha_{\bar{r}_{i}, \bar{s}_{i}}+\sum_{i} \bar{m}_{i} \alpha_{+} \sum_{i} \bar{n}_{i} \alpha_{-}=0
$$

For example for the 1-point correlation function

$$
\left\langle B(\alpha)\left|\phi_{(r, s ; \bar{r}, \bar{s})}(z, \bar{z})\right| 0,0 ; \alpha_{0}\right\rangle=\left\langle B(\alpha)\left|\mathcal{V}_{(r, s)}^{(m, n)}(z) \overline{\mathcal{V}}_{(r, s)}^{(\bar{m}, \bar{n})}(\bar{z})\right| 0,0 ; \alpha_{0}\right\rangle
$$
to

with $(\bar{r}, \bar{s})=(r, s)$. The combination of the equations (43) and (44) leads

$$
m=\bar{m}=n=\bar{n}=0
$$

So that in this case there is no screening operators and one find

$$
\alpha=\alpha_{r, s}
$$


Then we have

$$
\begin{aligned}
\left\langle B\left(\alpha_{r, s}\right)\left|\Phi_{(r, s ; \bar{r}, \bar{s})}(z, \bar{z})\right| 0,0 ; \alpha_{0}\right\rangle & =\left\langle B\left(\alpha_{r, s}\right)\left|\mathcal{V}_{(r, s)}(z) \overline{\mathcal{V}}_{(\bar{r}, \bar{s})}(\bar{z})\right| 0,0 ; \alpha_{0}\right\rangle \\
& =(1-z \bar{z})^{-2 h_{r, s}}
\end{aligned}
$$

Applying these algorithm for the Tricritical Ising model defined on the unit disk (UD) (i.e in the $\zeta-$ plane) one obtains

$$
\begin{aligned}
\langle I|I(\zeta, \bar{\zeta})| 0\rangle & =\left\langle I\left|\mathcal{V}_{1,1}(\zeta) \overline{\mathcal{V}}_{3,4}(\bar{\zeta})\right| 0,0 ; \alpha_{0}\right\rangle \\
& =\frac{1}{\sqrt[4]{5}} \sqrt{\frac{s_{2}}{2}}\left\langle B\left(\alpha_{1,1}\right)\left|\mathcal{V}_{1,1}(\zeta) \overline{\mathcal{V}}_{3,4}(\bar{\zeta})\right| 0,0 ; \alpha_{0}\right\rangle \\
& =\frac{1}{\sqrt[4]{5}} \sqrt{\frac{s_{2}}{2}}(1-\zeta \bar{\zeta})^{-2 h_{1,1}}=\frac{1}{\sqrt[4]{5}} \sqrt{\frac{s_{2}}{2}} \\
\langle\varepsilon|I(\zeta, \bar{\zeta})| 0\rangle & =\langle\varepsilon \mid 0\rangle=\frac{1}{\sqrt[4]{5}} \frac{s_{1}}{\sqrt{2 s_{2}}}, \\
\left\langle\varepsilon^{\prime}|I(\zeta, \bar{\zeta})| 0\right\rangle & =\left\langle\varepsilon^{\prime} \mid 0\right\rangle=\frac{1}{\sqrt[4]{5}} \frac{s_{1}}{\sqrt{2 s_{2}}}, \\
\left\langle\varepsilon^{\prime \prime}|I(\zeta, \bar{\zeta})| 0\right\rangle & =\left\langle\varepsilon^{\prime \prime} \mid 0\right\rangle=\frac{1}{\sqrt[4]{5}} \sqrt{\frac{s_{2}}{2}} \\
\langle\sigma|I(\zeta, \bar{\zeta})| 0\rangle & =\langle\sigma \mid 0\rangle=\frac{1}{\sqrt[4]{5}} \frac{s_{1}}{\sqrt{s_{2}}} \\
\left\langle\sigma^{\prime}|I(\zeta, \bar{\zeta})| 0\right\rangle & =\left\langle\sigma^{\prime} \mid 0\right\rangle=\frac{1}{\sqrt[4]{5}} \sqrt{s_{2}}
\end{aligned}
$$


For the operator $\varepsilon$

$$
\begin{aligned}
\langle I|\varepsilon(\zeta, \bar{\zeta})| 0\rangle & =\left\langle I\left|\mathcal{V}_{1,2}(\zeta) \overline{\mathcal{V}}_{3,3}(\bar{\zeta})\right| 0,0 ; \alpha_{0}\right\rangle \\
& =\frac{1}{\sqrt[4]{5}} \sqrt{\frac{s_{1}}{2}}\left\langle B\left(\alpha_{1,2}\right)\left|\mathcal{V}_{1,2}(\zeta) \overline{\mathcal{V}}_{3,3}(\bar{\zeta})\right| 0,0 ; \alpha_{0}\right\rangle \\
& =\frac{1}{\sqrt[4]{5}} \sqrt{\frac{s_{1}}{2}}(1-\zeta \bar{\zeta})^{-\frac{1}{5}} \\
\langle\varepsilon|\varepsilon(\zeta, \bar{\zeta})| 0\rangle & =-\frac{1}{\sqrt[4]{5}} \frac{s_{2}}{\sqrt{2 s_{1}}}(1-\zeta \bar{\zeta})^{-\frac{1}{5}} \\
\left\langle\varepsilon^{\prime}|\varepsilon(\zeta, \bar{\zeta})| 0\right\rangle & =-\frac{1}{\sqrt[4]{5}} \frac{s_{2}}{\sqrt{2 s_{1}}}(1-\zeta \bar{\zeta})^{-\frac{1}{5}}, \\
\left\langle\varepsilon^{\prime \prime}|\varepsilon(\zeta, \bar{\zeta})| 0\right\rangle & =\frac{1}{\sqrt[4]{5}} \sqrt{\frac{s_{1}}{2}}(1-\zeta \bar{\zeta})^{-\frac{1}{5}} \\
\langle\sigma|\varepsilon(\zeta, \bar{\zeta})| 0\rangle & =\frac{1}{\sqrt[4]{5}} \frac{s_{2}}{\sqrt{s_{1}}}(1-\zeta \bar{\zeta})^{-\frac{1}{5}} \\
\left\langle\sigma^{\prime}|\varepsilon(\zeta, \bar{\zeta})| 0\right\rangle & =-\frac{1}{\sqrt[4]{5}} \sqrt{s_{1}}(1-\zeta \bar{\zeta})^{-\frac{1}{5}}
\end{aligned}
$$

and for the spin operator $\sigma$

$$
\begin{aligned}
\langle I|\sigma(\zeta, \bar{\zeta})| 0\rangle & =\frac{1}{\sqrt[4]{5}} s_{1}(1-\zeta \bar{\zeta})^{-\frac{3}{40}} \\
\langle\varepsilon|\sigma(\zeta, \bar{\zeta})| 0\rangle & =\frac{1}{\sqrt[4]{10}} \frac{s_{2}}{\sqrt{s_{1}}}(1-\zeta \bar{\zeta})^{-\frac{3}{40}} \\
\left\langle\varepsilon^{\prime}|\sigma(\zeta, \bar{\zeta})| 0\right\rangle & =-\frac{1}{\sqrt[4]{10}} \frac{s_{2}}{\sqrt{s_{1}}}(1-\zeta \bar{\zeta})^{-\frac{3}{40}} \\
\left\langle\varepsilon^{\prime \prime}|\sigma(\zeta, \bar{\zeta})| 0\right\rangle & =-\frac{1}{\sqrt[4]{10}} \sqrt{s_{1}}(1-\zeta \bar{\zeta})^{-\frac{3}{40}} \\
\langle\sigma|\sigma(\zeta, \bar{\zeta})| 0\rangle & =0 \\
\left\langle\sigma^{\prime}|\sigma(\zeta, \bar{\zeta})| 0\right\rangle & =0
\end{aligned}
$$

At this level one can come back to the UHP by the global conformal transformation

$$
z=-i y_{0} \frac{\zeta-1}{\zeta+1} \quad, \quad \bar{z}=i y_{0} \frac{\bar{\zeta}-1}{\bar{\zeta}+1}
$$

which map the origin $\zeta=0$ to the point $z=i y_{0}$. The effect of this transfor- 
mation on $n$-point correlation functions is given by

$$
\begin{aligned}
& <\phi_{1}\left(z_{1}, \bar{z}_{1}\right) \phi_{2}\left(z_{2}, \bar{z}_{2}\right) \ldots \ldots \phi_{n}\left(z_{p}, \bar{z}_{p}\right)>_{U H P} \\
& =\prod_{i=1}^{n}\left(\frac{d z_{i}}{d \zeta_{i}}\right)^{-h_{i}}\left(\frac{d \bar{z}_{i}}{d \bar{\zeta}_{i}}\right)^{-h_{i}}<\phi_{1}\left(\zeta_{1}, \bar{\zeta}_{1}\right) \phi_{2}\left(\zeta_{2}, \bar{\zeta}_{2}\right) \ldots \ldots \phi_{n}\left(\zeta_{p}, \bar{\zeta}_{p}\right)>_{D i s k} \\
& =\prod_{i=1}^{n}\left(\frac{2 y_{0}}{\left(\zeta_{i}+1\right)\left(\bar{\zeta}_{i}+1\right)}\right)^{-2 h_{i}}<\phi_{1}\left(\zeta_{1}, \bar{\zeta}_{1}\right) \phi_{2}\left(\zeta_{2}, \bar{\zeta}_{2}\right) \ldots \ldots \phi_{n}\left(\zeta_{p}, \bar{\zeta}_{p}\right)>_{D i s k}
\end{aligned}
$$

For example the one point correlators given in equations (501) and (51) are transformed by the transformation (52) into

$$
\begin{aligned}
\langle\varepsilon(z, \bar{z})\rangle_{I} & =\frac{\left\langle I|\varepsilon(z, \bar{z})| 0,0 ; \alpha_{0}\right\rangle}{\left\langle I \mid 0,0 ; \alpha_{0}\right\rangle}=\left(\frac{s_{1}}{s_{2}}\right)^{\frac{1}{2}}(2 y)^{-\frac{1}{5}} \\
\langle\varepsilon(z, \bar{z})\rangle_{\varepsilon} & =-\left(\frac{s_{2}}{s_{1}}\right)^{\frac{3}{2}}(2 y)^{-\frac{1}{5}} \\
\langle\varepsilon(z, \bar{z})\rangle_{\varepsilon^{\prime}} & =-\left(\frac{s_{2}}{s_{1}}\right)^{\frac{3}{2}}(2 y)^{-\frac{1}{5}} \\
\langle\varepsilon(z, \bar{z})\rangle_{\varepsilon^{\prime \prime}} & =\left(\frac{s_{1}}{s_{2}}\right)^{\frac{1}{2}}(2 y)^{-\frac{1}{5}} \\
\langle\varepsilon(z, \bar{z})\rangle_{\sigma} & =\left(\frac{s_{2}}{s_{1}}\right)^{\frac{3}{2}}(2 y)^{-\frac{1}{5}} \\
\langle\varepsilon(z, \bar{z})\rangle_{\sigma^{\prime}} & =-\left(\frac{s_{1}}{s_{2}}\right)^{\frac{1}{2}}(2 y)^{-\frac{1}{5}} \\
\langle\sigma(z, \bar{z})\rangle_{I} & =\sqrt{2} \frac{s_{1}}{\sqrt{s_{2}}}(2 y)^{-\frac{3}{40}} \\
\langle\sigma(z, \bar{z})\rangle_{\varepsilon} & =\sqrt[4]{2}\left(\frac{s_{2}}{s_{1}}\right)^{\frac{3}{2}}(2 y)^{-\frac{3}{40}} \\
\langle\sigma(z, \bar{z})\rangle_{\varepsilon^{\prime}} & =-\sqrt[4]{2}\left(\frac{s_{2}}{s_{1}}\right)^{\frac{3}{2}}(2 y)^{-\frac{3}{40}} \\
\langle\sigma(z, \bar{z})\rangle_{\varepsilon^{\prime \prime}} & =-\sqrt[4]{2}\left(\frac{s_{1}}{s_{2}}\right)^{\frac{1}{2}}(2 y)^{-\frac{3}{40}} \\
\langle\sigma(z, \bar{z})\rangle_{\sigma} & =0 \\
\langle\sigma(z, \bar{z})\rangle_{\sigma^{\prime}} & =0
\end{aligned}
$$




\subsection{The boundary 2-point boundary correlation func- tions:}

We consider the two point function of the field $\Phi_{1,2} \equiv \varepsilon$. They are of the form

$$
\begin{aligned}
& \left\langle B(\alpha)\left|\Phi_{1,2}\left(\zeta_{1}, \bar{\zeta}_{1}\right) \Phi_{1,2}\left(\zeta_{2}, \bar{\zeta}_{2}\right)\right| 0,0 ; \alpha_{0}\right\rangle \\
= & \left\langle B(\alpha)\left|\mathcal{V}_{1,2}^{m_{1}, n_{1}}\left(\zeta_{1}\right) \overline{\mathcal{V}}_{\left(p^{\prime}-1, p-2\right)}^{\bar{m}_{1}, \bar{n}_{1}}\left(\bar{\zeta}_{1}\right) \mathcal{V}_{1,2}^{m_{2}, n_{2}}\left(\zeta_{2}\right) \overline{\mathcal{V}}_{1,2}^{\bar{m}_{2}, \bar{n}_{2}}\left(\bar{\zeta}_{2}\right)\right| 0,0 ; \alpha_{0}\right\rangle
\end{aligned}
$$

where we taked first $\Phi_{1,2}\left(\zeta_{1}, \bar{\zeta}_{1}\right) \sim \mathcal{V}_{1,2}\left(\zeta_{1}\right) \overline{\mathcal{V}}_{\left(p^{\prime}-1, p-2\right)}\left(\bar{\zeta}_{1}\right)$ and then $\Phi_{1,2}\left(\zeta_{1}, \bar{\zeta}_{1}\right) \sim$ $\mathcal{V}_{1,2}\left(\zeta_{2}\right) \overline{\mathcal{V}}_{1,2}\left(\bar{\zeta}_{2}\right)$

From the neutrality condition, we obtain the following constraints

$$
\begin{aligned}
\alpha & =\left(m_{1}+m_{2}\right) \alpha_{+}+\left(n_{1}+n_{2}-1\right) \alpha_{-}, \\
2 \alpha_{0}-\alpha & =\left(\bar{m}_{1}+\bar{m}_{2}+1\right) \alpha_{+}+\left(\bar{n}_{1}+\bar{n}_{2}+1\right) \alpha_{-}
\end{aligned}
$$

which can be solved as

$$
\begin{aligned}
m & =\bar{m}=\bar{n}=0, n=1, \\
m & =\bar{m}=n=0, \bar{n}=1
\end{aligned}
$$

with $m=m_{1}+m_{2}, n=n_{1}+n_{2}, \bar{m}=\bar{m}_{1}+\bar{m}_{2}, \bar{n}=\bar{n}_{1}+\bar{n}_{2}$.

The first solution (56) corresponds to $\alpha=\alpha_{1,1}=0$, and to the conformal block

$$
\begin{aligned}
I_{1}= & N_{1}\left(1-\zeta_{1} \bar{\zeta}_{1}\right)^{a}\left(1-\zeta_{2} \bar{\zeta}_{2}\right)^{a}[\eta(\eta-1)]^{a} \\
& \times \frac{\Gamma\left(1-\alpha_{-}^{2}\right)^{2}}{\Gamma\left(2-2 \alpha_{-}^{2}\right)} F\left(2 a, 1-\alpha_{-}^{2}, 2-2 \alpha_{-}^{2} ; \eta\right)
\end{aligned}
$$

with $a=2 \alpha_{1,2}\left(2 \alpha_{0}-\alpha_{1,2}\right), F$ being the hypergeometric function and $\eta$ is the parameter defined by

$$
\eta=\frac{\left(\zeta_{1}-\zeta_{2}\right)\left(\bar{\zeta}_{1}-\bar{\zeta}_{2}\right)}{\left(1-\zeta_{1} \bar{\zeta}_{1}\right)\left(1-\bar{\zeta}_{2} \bar{\zeta}_{2}\right)}
$$

The second solution (57) gives $\alpha=\alpha_{1,3}=-\alpha_{-}$, and corresponds to the conformal block

$$
\begin{aligned}
I_{2}= & N_{2}\left(1-\zeta_{1} \bar{\zeta}_{1}\right)^{a}\left(1-\zeta_{2} \bar{\zeta}_{2}\right)^{a}[\eta(\eta-1)]^{a}(-\eta)^{b-a} \\
& \times \frac{\Gamma\left(1-\alpha_{-}^{2}\right) \Gamma\left(3 \alpha_{-}^{2}-1\right)}{\Gamma\left(2 \alpha_{-}^{2}\right)} F\left(\alpha_{-}^{2}, 1-\alpha_{-}^{2}, 2 \alpha_{-}^{2} ; \eta\right)
\end{aligned}
$$


with $b=2 \alpha_{1,2}^{2}$.

Using (52) one can write these equations on the UHP as

$$
\begin{aligned}
I_{1}= & N_{1}\left\{\frac{\left(z_{1}-\bar{z}_{1}\right)\left(\bar{z}_{2}-z_{2}\right)}{\left(z_{1}-z_{2}\right)\left(\bar{z}_{1}-\bar{z}_{2}\right)\left(z_{1}-\bar{z}_{2}\right)\left(\bar{z}_{1}-z_{2}\right)}\right\}^{2 h_{1,2}} \\
& \times \frac{\Gamma\left(1-\alpha_{-}^{2}\right)^{2}}{\Gamma\left(2-2 \alpha_{-}^{2}\right)} F\left(-4 h_{1,2}, 1-\alpha_{-}^{2}, 2-2 \alpha_{-}^{2} ; \eta\right)
\end{aligned}
$$

and

$$
\begin{aligned}
I_{2}= & N_{2}\left\{\frac{\left(z_{1}-\bar{z}_{1}\right)\left(\bar{z}_{2}-z_{2}\right)}{\left(z_{1}-z_{2}\right)\left(\bar{z}_{1}-\bar{z}_{2}\right)\left(z_{1}-\bar{z}_{2}\right)\left(\bar{z}_{1}-z_{2}\right)}\right\}^{2 h_{1,2}} \\
& \times \frac{\Gamma\left(1-\alpha_{-}^{2}\right) \Gamma\left(3 \alpha_{-}^{2}-1\right)}{\Gamma\left(2 \alpha_{-}^{2}\right)} F\left(\alpha_{-}^{2}, 1-\alpha_{-}^{2}, 2 \alpha_{-}^{2} ; \eta\right)
\end{aligned}
$$

with

$$
\eta=\frac{\left(\zeta_{1}-\zeta_{2}\right)\left(\bar{\zeta}_{1}-\bar{\zeta}_{2}\right)}{\left(1-\zeta_{1} \bar{\zeta}_{1}\right)\left(1-\zeta_{2} \bar{\zeta}_{2}\right)} \rightarrow \frac{\left(z_{1}-z_{2}\right)\left(\bar{z}_{1}-\bar{z}_{2}\right)}{\left(z_{1}-\bar{z}_{1}\right)\left(z_{2}-\bar{z}_{2}\right)}
$$

Combining the two blocks with the coefficients appearing in the development of the boundary states we obtain for the correlators in the UHP

$$
\begin{aligned}
\left\langle\varepsilon\left(z_{1}, \bar{z}_{1}\right) \varepsilon\left(z_{2}, \bar{z}_{2}\right)\right\rangle_{\tilde{I}}= & \left\{\frac{\left(z_{1}-\bar{z}_{1}\right)\left(\bar{z}_{2}-z_{2}\right)}{\left(z_{1}-z_{2}\right)\left(\bar{z}_{1}-\bar{z}_{2}\right)\left(z_{1}-\bar{z}_{2}\right)\left(\bar{z}_{1}-z_{2}\right)}\right\}^{\frac{1}{5}} \times \\
& \left\{N_{1} \frac{\Gamma(1 / 5)^{2}}{\Gamma(2 / 5)} F_{1}+\sqrt{\frac{s_{1}}{s_{2}}} N_{2} \frac{\Gamma\left(\frac{1}{5}\right) \Gamma\left(\frac{7}{5}\right)}{\Gamma\left(\frac{8}{5}\right)} \times(-\eta)^{9 / 5} F_{2}\right\}, \\
\left\langle\varepsilon\left(z_{1}, \bar{z}_{1}\right) \varepsilon\left(z_{2}, \bar{z}_{2}\right)\right\rangle_{\widetilde{\varepsilon}}= & \left\{\frac{\left(z_{1}-\bar{z}_{1}\right)\left(\bar{z}_{2}-z_{2}\right)}{\left(z_{1}-z_{2}\right)\left(\bar{z}_{1}-\bar{z}_{2}\right)\left(z_{1}-\bar{z}_{2}\right)\left(\bar{z}_{1}-z_{2}\right)}\right\}^{\frac{1}{5}} \times \\
& \left\{N_{1} \frac{\Gamma(1 / 5)^{2}}{\Gamma(2 / 5)} F_{1}-\left(\frac{s_{2}}{s_{1}}\right)^{3 / 2} N_{2} \frac{\Gamma\left(\frac{1}{5}\right) \Gamma\left(\frac{7}{5}\right)}{\Gamma\left(\frac{8}{5}\right)} \times(-\eta)^{9 / 5} F_{2}\right\}, \\
\left\langle\varepsilon\left(z_{1}, \bar{z}_{1}\right) \varepsilon\left(z_{2}, \bar{z}_{2}\right)\right\rangle_{\widetilde{\varepsilon}^{\prime}}= & \left\{\frac{\left(z_{1}-\bar{z}_{1}\right)\left(\bar{z}_{2}-z_{2}\right)}{\left(z_{1}-z_{2}\right)\left(\bar{z}_{1}-\bar{z}_{2}\right)\left(z_{1}-\bar{z}_{2}\right)\left(\bar{z}_{1}-z_{2}\right)}\right\}^{\frac{1}{5}} \times \\
& \left\{N_{1} \frac{\Gamma(1 / 5)^{2}}{\Gamma(2 / 5)} F_{1}-\left(\frac{s_{2}}{s_{1}}\right)^{3 / 2} N_{2} \frac{\Gamma\left(\frac{1}{5}\right) \Gamma\left(\frac{7}{5}\right)}{\Gamma\left(\frac{8}{5}\right)} \times(-\eta)^{9 / 5} F_{2}\right\},
\end{aligned}
$$




$$
\begin{aligned}
\left\langle\varepsilon\left(z_{1}, \bar{z}_{1}\right) \varepsilon\left(z_{2}, \bar{z}_{2}\right)\right\rangle_{\widetilde{\varepsilon}^{\prime \prime}}= & \left\{\frac{\left(z_{1}-\bar{z}_{1}\right)\left(\bar{z}_{2}-z_{2}\right)}{\left(z_{1}-z_{2}\right)\left(\bar{z}_{1}-\bar{z}_{2}\right)\left(z_{1}-\bar{z}_{2}\right)\left(\bar{z}_{1}-z_{2}\right)}\right\}^{\frac{1}{5}} \times \\
& \left\{N_{1} \frac{\Gamma(1 / 5)^{2}}{\Gamma(2 / 5)} F_{1}+\left(\frac{s_{1}}{s_{2}}\right)^{1 / 2} N_{2} \frac{\Gamma\left(\frac{1}{5}\right) \Gamma\left(\frac{7}{5}\right)}{\Gamma\left(\frac{8}{5}\right)} \times(-\eta)^{9 / 5} F_{2}\right\}, \\
\left\langle\varepsilon\left(z_{1}, \bar{z}_{1}\right) \varepsilon\left(z_{2}, \bar{z}_{2}\right)\right\rangle_{\widetilde{\sigma}}= & \left\{\frac{\left(z_{1}-\bar{z}_{1}\right)\left(\bar{z}_{2}-z_{2}\right)}{\left(z_{1}-z_{2}\right)\left(\bar{z}_{1}-\bar{z}_{2}\right)\left(z_{1}-\bar{z}_{2}\right)\left(\bar{z}_{1}-z_{2}\right)}\right\}^{\frac{1}{5}} \times \\
& \left\{N_{1} \frac{\Gamma(1 / 5)^{2}}{\Gamma(2 / 5)} F_{1}-\left(\frac{s_{2}}{s_{1}}\right)^{3 / 2} N_{2} \frac{\Gamma\left(\frac{1}{5}\right) \Gamma\left(\frac{7}{5}\right)}{\Gamma\left(\frac{8}{5}\right)} \times(-\eta)^{9 / 5} F_{2}\right\}, \\
\left\langle\varepsilon\left(z_{1}, \bar{z}_{1}\right) \varepsilon\left(z_{2}, \bar{z}_{2}\right)\right\rangle_{\widetilde{\sigma}^{\prime}}= & \left\{\frac{\left(z_{1}-\bar{z}_{1}\right)\left(\bar{z}_{2}-z_{2}\right)}{\left(z_{1}-z_{2}\right)\left(\bar{z}_{1}-\bar{z}_{2}\right)\left(z_{1}-\bar{z}_{2}\right)\left(\bar{z}_{1}-z_{2}\right)}\right\}^{\frac{1}{5}} \times \\
& \left\{N_{1} \frac{\Gamma(1 / 5)^{2}}{\Gamma(2 / 5)} F_{1}+\left(\frac{s_{1}}{s_{2}}\right)^{1 / 2} N_{2} \frac{\Gamma\left(\frac{1}{5}\right) \Gamma\left(\frac{7}{5}\right)}{\Gamma\left(\frac{8}{5}\right)} \times(-\eta)^{9 / 5} F_{2}\right\}
\end{aligned}
$$

with $F_{1}=F\left(-\frac{4}{10}, \frac{1}{5}, \frac{2}{5} ; \eta\right)$, et $F_{2}=F\left(\frac{4}{5}, \frac{1}{5}, \frac{8}{5} ; \eta\right)$.

\section{Conclusion}

We have obtained the boundary consistent states and the exact correlation functions of the tricritical Ising model, using the Coulomb-gas formalism. The results obtained for 1-point correlators are in concordance with that already known (see for example chapter 15 of [16] ).

We hope also to generalize the use of this method to the case of the nondiagonal minimal models, such as the one representing the tricritical 3states Potts model.

\section{Acknowledgments}

S.B. would like to thank T. Wydro and J.F. McCabe for introducing him to the computational methods used in conformal field theories and statistical physics, and T. Wydro for the warm hospitality during the stay at the university Paul Verlaine of Metz. 


\section{References}

[1] J.L. Cardy, Nucl. Phys. B 240 (1984) 514

[2] J. L. Cardy, Nucl. Phys. B 324 (1989) 581

[3] J.L. Cardy, Nucl. Phys. B 275 (1986) 200

[4] J.L. Cardy and D.C. Lewellen, Phys. Lett. B 259 (1991) 274

[5] S. Kawai, Coulomb-gas approach for boundary conformal field theory, Nucl. Phys. B630 (2002) 203221, [hep-th/0201146].

[6] S. Kawai, Free-field realisation of boundary states and boundary correlation functions of minimal models, J. Phys. A36 (2003) 6547, hep-th/0210032.

[7] J. Schulze, Nucl. Phys. B 489 (1997) 580

[8] D. Friedan, Z. Qiu and S. Shenker, superconformal invariance in two dimensions and the tricritical Ising model, Phys. Lett. 151B, 37 (1984).

[9] R. I. Nepomechie, Consistent superconformal boundary states, J. Phys. A 34 6509-6524, 2001. hep-th/0102010

[10] R. I. Nepomechie, Supersymmetry in the boundary tricritical Ising field theory, Int. J. Mod. Ohys. A17 3809, 2002.hep-th/0203123

[11] N. Ishibashi, The Boundary and Crosscap States in Conformal Field Theories, Mod. Phys. Lett. A 4 (1987) 251-264.

[12] J.-B. Zuber , CFT, BCFT, ADE and all that, Proceedings of the bariloche Summer School, "Quantum Symmetries in Theoretical Physics and Mathematics", Jan 2000, eds R. Coquereaux, A. Garcia and R. Trinchero, hep-th/0006151.

[13] V. B. Petkova and J.-B. Zuber , "Conformal Boundary Conditions and what they teach us", hep-th/0103007. Proceedings of Budapest School and Conferences. Nonperturbative Quantum Field Theoretic Methods and their Aplications, August 2000, World Scientific Publishing Company.

[14] E. Verlinde, Nucl. Phys. B300 [FS22] (1988) 360.

[15] P. Di Francesco, P. Mathieu and D. Sénechal, Conformal Field Theory, Springer Verlag New York 1997. 
[16] M. Henkel, Conformal Invariance and Critical Phenomena, Springer Berlin 1999. 